\title{
SOLUCIÓN LOCAL Y SINGULARIDAD PARA UNA ECUACIÓN DE VIGA NO LINEAL DE TIPO KIRCHHOFF CON TÉRMINO DISIPATIVO
}

\author{
Teófanes Quispe*
}

Resumen: En el presente trabajo, consideramos un problema mixto para una ecuación de viga no lineal de tipo Kirchhoff con término disipativo en un dominio acotado. Probamos la existencia y unicidad de soluciones locales con argumentos del punto fijo de Banach. También obtenemos la propiedad de singularidad en tiempo finito de las soluciones y las estimativas para el tiempo de explosión.

Palabras clave: Solución local, Ecuación de viga no lineal de tipo Kirchhoff, Método de Galerkin, Método del punto fijo, Singularidad de soluciones.

\section{LOCAL SOLUTION AND BLOW-UP FOR A NONLINEAR BEAM EQUATION OF KIRCHHOFF TYPE WITH DISSIPATIVE TERM}

\begin{abstract}
In present work, we considered a mixed problem for a nonlinear beam equation of Kirchhoff type with dissipative term in a bounded domain. We prove the existence and uniqueness of local solutions with arguments of the fixed point of Banach. Also we obtain the blow-up properties in finite time of solutions and the estimates for the explosion time.
\end{abstract}

Key words: Local solution, Nonlinear beam equation of Kirchhoff type, Galerkin method, Fixed point method, Blow-up of solutions.

\section{INTRODUCCIÓN}

En este artículo consideramos el problema de valor inicial y de frontera para la siguiente ecuación de viga no lineal de tipo Kirchhoff:

$$
\left.u^{\prime \prime}+\beta(x) u^{\prime}+\alpha \Delta^{2} u-M\left(|\nabla u|_{2}^{2}\right) \Delta u=f(u) \text { en } \Omega \times\right] 0, \infty[
$$

con condiciones iniciales

$$
u(x, 0)=u_{0}(x), u^{\prime}(x, 0)=u_{1}(x), \text { en } \Omega
$$

y condiciones de frontera

$$
\left.u=\frac{\partial u}{\partial \nu}=0, \text { en } \partial \Omega \times\right] 0, \infty[
$$

donde $\Omega$ es un conjunto abierto y acotado de $\mathbb{R}^{n}$ con frontera suficientemente regular $\partial \Omega, \nabla$ es el operador gradiente, $\Delta$ es el operador laplaciano, $\alpha$ es una constante real positiva, $\beta(x)$ es una función real no negativa para $x \in \Omega, M(s)$ es una función real positiva de clase $C^{1}$ para $s \geq 0, f(s)$ es una función real no lineal continuamente diferenciable para $s \in \mathbb{R}, \frac{\partial}{\partial \nu}$ es la derivada normal sobre $\partial \Omega, w^{\prime}:=\frac{\partial w}{\partial t}$, $w^{\prime \prime}:=\frac{\partial^{2} w}{\partial t^{2}} \mathrm{y}|\nabla w|_{2}^{2}:=\int_{\Omega}|\nabla w(x, t)|^{2} d x$.

El caso $n=1$, la ecuación (1.1) describe vibraciones transversales no lineales de una viga de longitud $L$ de material elástico, cuyos extremos se encuentran fijos en los puntos $x=0$ y $x=L$, en el eje $x$ del plano $x u$. En estas condiciones la ecuación resultante es

$$
\rho h u^{\prime \prime}+\beta u^{\prime}+E I \frac{\partial^{4} u}{\partial x^{4}}-\left(p_{o}+\frac{E h}{2 L}\left|\frac{\partial u}{\partial x}\right|_{2}^{2}\right) \frac{\partial^{2} u}{\partial x^{2}}=f(u)
$$

*UNMSMS, Facultad de Ciencias Matemáticas, e-mail: tquispem@unmsm.edu.pe 
donde $u=u(x, t)$ representa el desplazamiento transversal en el espacio de coordenada $x$ y en el tiempo $t, \rho$ es la densidad de masa, $h$ es el área de la sección transversal de la viga, $p_{0}$ es la tensión inicial, $E$ es el módulo de Young del material, $I$ es el momento de inercia, el producto $E I$ es el módulo de rigidez de flexibilidad, $\beta$ es el coeficiente de la fuerza amortiguadora y $f(u)$ es la fuerza restauradora. Cuando en (1.4), $\beta \equiv f \equiv 0$ se tiene que la ecuación (1.4) es la propuesta y estudiada por Woinowsky-Krieger [16] y cuando $\beta \equiv I \equiv 0$ es la propuesta y estudiada por Kirchhoff [8]. El caso general $n \geq 1$, la ecuación (1.1) tiene diversas aplicaciones, como en el área de resistencia de materiales, mecánica de sólidos, análisis de estructuras, entre otras.

Cuando $\beta \equiv f \equiv 0$, el modelo (1.4) fue investigado por Eisley [6], mientras los resultados experimentales han sido dados por Burgreen [3]. También el modelo fue estudiado por Dickey [5] y más tarde por Ball [2]. Cuando $\beta \equiv f \equiv 0$, la ecuación (1.1) fue investigado por Tucsnak [14], quien obtiene decaimiento exponencial. Cuando $f \equiv 0, M \geq 0$ y término disipativo relativamente general, la ecuación (1.1) fue estudiado por Vasconsellos y Teixeira [15], quienes obtienen existencia, unicidad y decaimiento de la energía. Cuando $\beta \equiv 0$, la ecuación (1.1) fue también estudiado por Guedda y Labani [7], quienes obtienen no existencia global con energía inicial no positiva; por su parte Wu y Tsai [17], obtienen existencia local, existencia global y singularidad para $f(u)=|u|^{p-2} u, p>2$.

En este trabajo, probaremos la existencia, unicidad y propiedad de singularidad de las soluciones locales del problema (1.1) - (1.3) en un dominio acotado $\Omega$ en $\mathbb{R}^{n}$, cuando $\beta$ es una función real no negativa, $\alpha$ es una constante real positiva, $M$ es una función continua positiva y $f$ es una función real no lineal. En primer lugar, probaremos la existencia y la unicidad de la solución global de un problema lineal asociado a (1.1) - (1.3), utilizando el método de Galerkin y el método de la energía respectivamente. Asimismo, obtendremos una estimativa para las correspondientes soluciones. En segundo lugar, li-nealizaremos el problema (1.1) - (1.3) para elementos de un espacio $G_{T_{0}, R_{0}}$, llamado espacio de soluciones, luego obtendremos sus soluciones en $G_{T_{0}, R_{0}}$ y después, por argumentos del teorema de punto fijo de Banach, se obtendran las soluciones locales del problema (1.1) - (1.3) sobre un intervalo $\left[0, T_{0}\right]$, donde $T_{0}>0$ depende de los datos iniciales y de los parámetros del problema. La unicidad de soluciones será obtenida utilizando las estimativas de las soluciones del problema lineal. En tercer lugar, obtendremos la singularidad en tiempo finito de soluciones, con energía inicial negativa, nula y positiva restringida, empleando el método directo [9]. Asimismo hallaremos las estimativas para el tiempo finito de explosión. De esta manera, se puede extender en [17] a una fuerza restauradora relativamente más general $f(u)$ y adicionar el término disipativo lineal $\beta(x) u^{\prime}$. En la discusión del problema emplearemos las estrategias y herramientas inspiradas en los trabajos de Li y Tsai [9], Wu y Tsai [17] y Quispe Méndez [10,11, 12, 13].

\section{PRELIMINARES}

En esta sección, presentamos algunas notaciones, conceptos y resultados sin demostración, los cuales serán usados en el desarrollo del presente trabajo.

Sea $\Omega$ un conjunto abierto y acotado de $\mathbb{R}^{n}$ con frontera suficientemente regular $\partial \Omega$. Denotamos el producto interno y la norma de $L^{2}(\Omega)$ y $L^{p}(\Omega)$, con $(.,$.$) y \mid$. $\left.\right|_{p}$ respectivamente, para $1 \leq p \leq \infty$. Además $((.,)$.$) y \|$.$\| , denotaran el producto interno y la norma de H_{0}^{1}(\Omega)$, donde $((u, v)):=\int_{\Omega} \nabla u(x) . \nabla v(x) d x$ es la forma de Dirichlet.

Sea $X$ un espacio de Banach, $T$ y $p$ números reales tales que $0<T \leq \infty$ y $1 \leq p \leq \infty$. Representamos con $L^{p}(0, T ; X)$ al espacio de Banach de las funciones vectoriales $\left.u:\right] 0, T[\longrightarrow X$ medibles con $\|u(t)\|_{X} \in L^{p}(0, T)$, dotado de la norma

$$
\begin{gathered}
\|u\|_{L^{p}(0, T ; X)}:=\left(\int_{0}^{T}\|u(t)\|_{X}^{p} d t\right)^{\frac{1}{p}}, 1 \leq p<\infty \\
\|u\|_{L^{\infty}(0, T ; X)}:=\sup _{0<t<T} \operatorname{ess}\|u(t)\|_{X}, p=\infty .
\end{gathered}
$$


Similarmente, cuando $0<T<\infty$, representamos con $C([0, T] ; X)$ al espacio de Banach de las funciones continuas $u:[0, T] \longrightarrow X$, dotado de la norma

$$
\|u\|_{C([0, T] ; X)}:=\sup _{0 \leq t \leq T}\|u(t)\|_{X} .
$$

Denotamos $w^{\prime}:=\frac{\partial w}{\partial t}, w^{\prime \prime}:=\frac{\partial^{2} w}{\partial t^{2}}, w(t)(x):=w(x, t)$ y $L^{\infty}:=L^{\infty}(\Omega)$.

Hipótesis. Imponemos sobre las funciones reales $\mu(t), \beta(x), M(s)$ y $f(s)$ las siguientes condiciones:

(H1) $\mu \in C\left(\left[0, \infty[), \mu^{\prime} \in L^{2}(0, \infty)\right.\right.$ y $\mu(t) \geq m_{0}>0, \forall t \geq 0$, donde $m_{0}$ es una constante positiva.

(H2) $\beta \in L^{\infty}(\Omega), \beta(x) \geq 0, \forall x \in \Omega$.

(H3) $M \in C^{1}\left(\left[0, \infty[)\right.\right.$ y $M(s) \geq m_{0}>0, \forall s \geq 0$, donde $m_{0}$ es una constante dada en $(H 1)$.

(H4) $f(0)=0$ y existe una constante positiva $K$ tal que

$$
|f(s)-f(r)| \leq K|s-r|\left(|s|^{p-2}+|r|^{p-2}\right),
$$

para $s, r \in \mathbb{R}$ y $2<p \leq \frac{2(n-3)}{n-4}$ para $n \geq 5$ ó $p>2$ para $n \leq 4$.

$(H 5)(2 \gamma+1) \widehat{M}(s) \geq\left(M(s)+2 \gamma m_{0}\right) s, \forall s \geq 0$, donde $\widehat{M}(s):=\int_{0}^{s} M(\xi) d \xi, 0<\gamma \leq \frac{p-2}{4}$ es una constante y $m_{0}$ es una constante dada en $(H 1)$.

(H6) $f(s) s \geq 2(2 \gamma+1) F(s), \forall s \in \mathbb{R}$, donde $F(s):=\int_{0}^{s} f(\xi) d \xi$ y $\gamma$ es una constante dada en (H5).

Lema 2.1 (Desigualdad de Sobolev-Poincaré [1]). Si $1 \leq p \leq \frac{2 n}{[n-2 m]^{+}}(1 \leq p<\infty$ si $n=2 m)$, entonces existe una constante positiva $B_{1}$ tal que

$$
|u|_{p} \leq B_{1}\left|(-\Delta)^{\frac{m}{2}} u\right|_{2}, \forall u \in D\left((-\Delta)^{\frac{m}{2}}\right)
$$

donde $[a]^{+}:=\operatorname{máx}\{0, a\}$ y $\frac{1}{[a]^{+}}=\infty$ si $[a]^{+}=0$.

Lema 2.2 (Desigualdad de Gronwall [18]). Sean $f, g:[a, b] \rightarrow \mathbb{R}$ funciones continuas, con $g$ no decreciente y $h \in L^{1}(a, b)$, las cuales satisfacen la desigualdad

$$
f(t) \leq g(t)+\int_{a}^{t} h(s) f(s) d s, \forall t \in[a, b] .
$$

Entonces,

$$
f(t) \leq g(t) \exp \left(\int_{a}^{t} h(s) d s\right), \forall t \in[a, b]
$$

Lema 2.3 ([9]). Sea $\gamma>0$ y sea $B \in C^{2}([0, \infty[)$ una función no negativa que satisface

$$
B^{\prime \prime}(t)-4(\gamma+1) B^{\prime}(t)+4(\gamma+1) B(t) \geq 0
$$

Si $B^{\prime}(0)>r_{2} B(0)+K_{0}$, entonces $B^{\prime}(t)>K_{0}$, para $t>0$, donde $K_{0}$ es una constante $y$

$$
r_{2}:=2(\gamma+1)-2 \sqrt{(\gamma+1) \gamma}
$$

es la menor raíz de la ecuación cuadrática $r^{2}-4(\gamma+1) r+4(\gamma+1)=0$.

Lema $2.4([9])$. Si $J(t)$ es una función no creciente en $\left[t_{0}, \infty\left[, t_{0} \geq 0\right.\right.$ y satisface la inecuación diferencial

$$
\left[J^{\prime}(t)\right]^{2} \geq a+b[J(t)]^{2+\frac{1}{\gamma}}, \text { para } t \geq t_{0},
$$

donde $a>0, \gamma>0$ y $b \in \mathbb{R}$, entonces existe un número real positivo $T_{*}$ tal que $\lim _{t \rightarrow T_{*}^{-}} J(t)=0$ y una cota superior de $T_{*}$ puede ser estimado respectivamente, en los siguientes casos: 
(i) Si $b<0$ y $J\left(t_{0}\right)<\min \left\{1, \sqrt{\frac{a}{-b}}\right\}$, entonces

$$
T_{*} \leq t_{0}+\frac{1}{\sqrt{-b}} \ln \left(\frac{\sqrt{\frac{a}{-b}}}{\sqrt{\frac{a}{-b}}-J\left(t_{0}\right)}\right) .
$$

(ii) Si $b=0$, entonces

$$
T_{*} \leq t_{0}+\frac{J\left(t_{0}\right)}{\sqrt{a}} .
$$

(iii) Si $b>0$, entonces

$$
T_{*} \leq \frac{J\left(t_{0}\right)}{\sqrt{a}}
$$

0

$$
T_{*} \leq t_{0}+2^{\frac{3 \gamma+1}{2 \gamma}} \frac{\gamma c}{\sqrt{a}}\left(1-\left[1+c J\left(t_{0}\right)\right]^{-\frac{1}{2 \gamma}}\right) \text {, }
$$

donde $c:=\left(\frac{b}{a}\right)^{\frac{\gamma}{\gamma+2}}$.

\section{PROBLEMA LINEAL}

En esta sección, estudiaremos la existencia y la unicidad de la solución global del siguiente problema lineal mixto:

$$
\begin{array}{ll}
u^{\prime \prime}+\beta(x) u^{\prime}+\alpha \Delta^{2} u-\mu(t) \Delta u=h(x, t) & \text { en } \Omega \times] 0, T[, \\
u(x, 0)=u_{0}(x), u^{\prime}(x, 0)=u_{1}(x) & \text { en } \Omega, \\
u=\frac{\partial u}{\partial \nu}=0 & \text { en } \partial \Omega \times] 0, T[
\end{array}
$$

donde $T$ es un número real positivo fijo, escogido arbitrariamente. En la discusión de la existencia, emplearemos el método de Galerkin y en la unicidad, el método de la energía.

Lema 3.1. Supongamos que las funciones $\mu$ y $\beta$ satisfacen las hipótesis (H1) y (H2) respectivamente, $u_{0} \in U, u_{1} \in H_{0}^{1}(\Omega) \cap H^{2}(\Omega)$ y $h \in W^{1,2}\left(0, T ; L^{2}(\Omega)\right)$. Entonces el problema (3.1) admite solución única u sobre $[0, T]$ tal que

$$
\begin{aligned}
& u \in L^{\infty}([0, T] ; U) \\
& u^{\prime} \in L^{\infty}\left(0, T ; H_{0}^{1}(\Omega) \cap H^{2}(\Omega)\right), \\
& u^{\prime \prime} \in L^{\infty}\left(0, T ; L^{2}(\Omega)\right)
\end{aligned}
$$

donde

$$
U:=\left\{u \in H_{0}^{2}(\Omega) ; \Delta^{2} u \in L^{2}(\Omega)\right\} .
$$

Además, la solución u verifica la estimativa

$$
E_{1}(t) \leq\left[E_{1}^{\frac{1}{2}}(0)+\int_{0}^{t}|h(s)|_{2} d s\right]^{2} \exp \left(\int_{0}^{t}\left[\frac{\left|\mu^{\prime}(s)\right|}{\mu(s)}+|\beta|_{L^{\infty}}\right] d s\right)
$$

para todo $t \in[0, T]$, donde

$$
\begin{aligned}
& E_{1}(t):=\frac{1}{2}\left|u^{\prime}(t)\right|_{2}^{2}+\frac{1}{2} \alpha|\Delta u(t)|_{2}^{2}+\frac{1}{2} \mu(t)\|u(t)\|^{2}, \\
& E_{1}(0):=\frac{1}{2}\left|u_{1}\right|_{2}^{2}+\frac{1}{2} \alpha\left|\Delta u_{0}\right|_{2}^{2}+\frac{1}{2} \mu(0)\left\|u_{0}\right\|^{2} .
\end{aligned}
$$

Demostración. Procedemos en cinco etapas.

Soluciones Aproximadas. Sea $\left\{\omega_{k}\right\}_{k \in \mathbb{N}}$ una base en el espacio $U$ y sea $V_{m}=\left[\omega_{1}, \omega_{2}, \ldots, \omega_{m}\right]$ el subespacio generado por los primeros $m$ vectores $\omega_{1}, \omega_{2}, \ldots, \omega_{m}$ de $\left\{\omega_{k}\right\}_{k \in \mathbb{N}}$. Consideremos 


$$
u_{m}(t)=\sum_{j=1}^{m} r_{j m}(t) \omega_{j},
$$

las soluciones aproximadas en $V_{m}$ del problema (3.1), donde las funciones $r_{j m}(t), j=1,2, \ldots, m$, son determinadas del siguiente problema en ecuaciones diferenciales ordinarias, para $w \in V_{m}$

$$
\begin{aligned}
& \left(u_{m}^{\prime \prime}(t), w\right)+\left(\beta u_{m}^{\prime}(t), w\right)+\alpha\left(\Delta u_{m}(t), \Delta w\right) \\
& +\mu(t)\left(\left(u_{m}(t), w\right)\right)=(h(t), w), \\
& u_{m}(0)=u_{0 m}, u_{m}^{\prime}(0)=u_{1 m},
\end{aligned}
$$

donde

$$
\begin{aligned}
& u_{0 m}=\sum_{j=1}^{m} r_{0 j m} w_{j}, \quad u_{0 m} \rightarrow u_{0} \text { fuerte en } U, \\
& u_{1 m}=\sum_{j=1}^{m} r_{1 j m} w_{j}, \quad u_{1 m} \rightarrow u_{1} \text { fuerte en } H_{0}^{1}(\Omega) \cap H^{2}(\Omega) .
\end{aligned}
$$

El teorema de Carathéodory [4], nos garantiza la existencia de una solución local $u_{m}$ del problema aproximado (3.3) en el intervalo $\left[0, T_{m}\left[, 0<T_{m}<T\right.\right.$. Las siguientes estimativas a priori nos permitirán extender la $u_{m}$ a todo el intervalo $[0, T]$, con $T$ independiente de $m$.

Estimativa I. Considerando $w=u_{m}^{\prime}(t)$ en (3.3), se obtiene

$$
\begin{aligned}
\frac{1}{2} \frac{d}{d t}\left|u_{m}^{\prime}(t)\right|_{2}^{2}+\left(\beta u_{m}^{\prime}(t), u_{m}^{\prime}(t)\right) & +\frac{1}{2} \alpha \frac{d}{d t}\left|\Delta u_{m}(t)\right|_{2}^{2} \\
+ & \frac{1}{2} \mu(t) \frac{d}{d t}\left\|u_{m}(t)\right\|^{2}=\left(h(t), u_{m}^{\prime}(t)\right) .
\end{aligned}
$$

Utilizando la desigualdad de Hölder y la hipótesis $(H 2)$, del resultado (3.5), se obtiene

$$
\frac{d}{d t} E_{1}(t) \leq|h(t)|_{2}\left|u_{m}^{\prime}(t)\right|_{2}+\frac{1}{2}\left|\mu^{\prime}(t)\right|\left\|u_{m}(t)\right\|^{2}+|\beta|_{L^{\infty}}\left|u_{m}^{\prime}(t)\right|_{2}^{2},
$$

donde

De (3.6), resulta

$$
E_{1}(t):=\frac{1}{2}\left|u_{m}^{\prime}(t)\right|_{2}^{2}+\frac{1}{2} \alpha\left|\Delta u_{m}(t)\right|_{2}^{2}+\frac{1}{2} \mu(t)\left\|u_{m}(t)\right\|^{2}
$$

$$
\frac{d}{d t} E_{1}^{\frac{1}{2}}(t) \leq|h(t)|_{2}+\frac{1}{2}\left[\frac{\left|\mu^{\prime}(t)\right|}{\mu(t)}+|\beta|_{L^{\infty}}\right] E_{1}^{\frac{1}{2}}(t) .
$$

Luego de integrar (3.7) desde 0 hasta $t$, y usando la desigualdad de Gronwall, se obtiene

$$
E_{1}(t) \leq\left[E_{1}^{\frac{1}{2}}(0)+\int_{0}^{t}|h(s)|_{2} d s\right]^{2} \exp \left(\int_{0}^{t}\left[\frac{\left|\mu^{\prime}(s)\right|}{\mu(t)}+|\beta|_{L^{\infty}}\right] d s\right),
$$

donde

$$
E_{1}(0):=\frac{1}{2}\left|u_{1 m}\right|_{2}^{2}+\frac{1}{2} \alpha\left|\Delta u_{0 m}\right|_{2}^{2}+\frac{1}{2} \mu(0)\left\|u_{0 m}\right\|^{2} .
$$

Por la condición (3.4) implica que $E_{1}$ (0) es acotado. Por las hipótesis de $h, \mu$ y $\beta$, del resultado (3.8), se tiene

$$
\left|u_{m}^{\prime}(t)\right|_{2}^{2}+\left|\Delta u_{m}(t)\right|_{2}^{2}+\left\|u_{m}(t)\right\|^{2} \leq C_{1}, \forall t \in[0, T]
$$

donde $C_{1}$ es una constante positiva independiente de $m$.

Estimativa II. Hacemos $t=0$ en (3.3) y luego tomando $w=u_{m}^{\prime \prime}(0)$, se obtiene

$$
\left|u_{m}^{\prime \prime}(0)\right|_{2}^{2} \leq\left|u_{m}^{\prime \prime}(0)\right|_{2}\left[|\beta|_{L^{\infty}}\left|u_{1 m}\right|_{2}+\alpha\left|\Delta^{2} u_{0 m}\right|_{2}+\mu(0)\left|\Delta u_{0 m}\right|_{2}+|h(0)|_{2}\right] .
$$

Así, usando las hipótesis de $h, \mu$ y $\beta$, y la condición (3.4), se tiene

$$
\left|u_{m}^{\prime \prime}(0)\right|_{2}^{2} \leq C_{2}
$$


donde $C_{2}$ es una constante positiva independiente de $m$.

Estimativa III. Tomando la derivada de (3.3) con respecto a $t$ y luego considerando $w=u_{m}^{\prime \prime}(t)$, se obtiene

$$
\begin{aligned}
& \frac{1}{2} \frac{d}{d t}\left|u_{m}^{\prime \prime}(t)\right|_{2}^{2}+\left(\beta u_{m}^{\prime \prime}(t), u_{m}^{\prime \prime}(t)\right)+\frac{1}{2} \alpha \frac{d}{d t}\left|\Delta u_{m}^{\prime}(t)\right|_{2}^{2} \\
& +\frac{1}{2} \mu(t) \frac{d}{d t}\left\|u_{m}^{\prime}(t)\right\|^{2}+\mu^{\prime}(t)\left(\left(u_{m}(t), u_{m}^{\prime \prime}(t)\right)\right)=\left(h^{\prime}(t), u_{m}^{\prime \prime}(t)\right) .
\end{aligned}
$$

Utilizando la desigualdad de Hölder, la desigualdad de Yaung y las hipótesis de $h, \mu$ y $\beta$, del resultado (3.11), se obtiene

$$
\begin{aligned}
\frac{d}{d t} E_{2}(t) \leq & \frac{1}{2}\left[1+\left|\mu^{\prime}(t)\right|+2|\beta|_{L^{\infty}}\right]\left|u_{m}^{\prime \prime}(t)\right|_{2}^{2}+\frac{1}{2}\left|\mu^{\prime}(t)\right|\left\|u_{m}^{\prime}(t)\right\|^{2} \\
& +\frac{1}{2}\left|\mu^{\prime}(t)\right|\left|\Delta u_{m}(t)\right|_{2}^{2}+\frac{1}{2}\left|h^{\prime}(t)\right|_{2}^{2}, 3.12
\end{aligned}
$$

donde

$$
E_{2}(t):=\frac{1}{2}\left|u_{m}^{\prime \prime}(t)\right|_{2}^{2}+\frac{1}{2} \alpha\left|\Delta u_{m}^{\prime}(t)\right|_{2}^{2}+\frac{1}{2} \mu(t)\left\|u_{m}^{\prime}(t)\right\|^{2}
$$

Utilizando (3.9), del resultado (3.12), se tiene

$$
\begin{aligned}
\frac{d}{d t} E_{2}(t) \leq & {\left[1+\left|\mu^{\prime}(t)\right|+\frac{\left|\mu^{\prime}(t)\right|}{\mu(t)}+2|\beta|_{L^{\infty}}\right] E_{2}(t) 3.14 } \\
& +\frac{1}{2}\left[C_{1}\left|\mu^{\prime}(t)\right|+\left|h^{\prime}(t)\right|_{2}^{2}\right] .
\end{aligned}
$$

Después de integrar (3.14) desde 0 hasta $t$ y usando (3.10), se obtiene

$$
E_{2}(t) \leq E_{C_{2}}+g(t)+\int_{0}^{t}\left[1+\left|\mu^{\prime}(s)\right|+\frac{\left|\mu^{\prime}(s)\right|}{\mu(s)}+2|\beta|_{L^{\infty}}\right] E_{2}(s) d s
$$

donde

$$
\begin{gathered}
E_{C_{2}}:=\frac{1}{2} C_{2}+\frac{1}{2} \alpha\left|\Delta u_{1 m}\right|_{2}^{2}+\frac{1}{2} \mu(0)\left\|u_{1 m}\right\|^{2}, \\
g(t):=\frac{1}{2} \int_{0}^{t}\left[C_{1}\left|\mu^{\prime}(s)\right|+\left|h^{\prime}(s)\right|_{2}^{2}\right] d s .
\end{gathered}
$$

Aplicando la desigualdad de Gronwall en (3.15), se obtiene

$$
E_{2}(t) \leq\left[E_{C_{2}}+g(t)\right] \exp \left(\int_{0}^{t}\left[1+\left|\mu^{\prime}(s)\right|+\frac{\left|\mu^{\prime}(s)\right|}{\mu(s)}+2|\beta|_{L^{\infty}}\right] d s\right) .
$$

Por la condición (3.4), resulta que $E_{C_{2}}$ es acotado. Utilizando las hipótesis de $h, \mu \mathrm{y} \beta$, del resultado (3.17), se tiene

$$
\left|u_{m}^{\prime \prime}(t)\right|_{2}^{2}+\left|\Delta u_{m}^{\prime}(t)\right|_{2}^{2}+\left\|u_{m}^{\prime}(t)\right\|^{2} \leq C_{3}, \forall t \in[0, T]
$$

donde $C_{3}$ es una constante positiva independiente de $m$.

Pasaje al límite. De las estimativas (3.9) y (3.18), existen subsucesiones $\left\{u_{\nu}\right\},\left\{u_{\nu}^{\prime}\right\}$ y $\left\{u_{\nu}^{\prime \prime}\right\}$ de $\left\{u_{m}\right\}$, $\left\{u_{m}^{\prime}\right\}$ y $\left\{u_{m}^{\prime \prime}\right\}$ respectivamente, tales que

$$
\begin{array}{lll}
u_{\nu} \stackrel{*}{*} u & \text { en } & L^{\infty}\left(0, T ; H_{0}^{2}(\Omega)\right), \\
u_{\nu}^{\prime} \stackrel{*}{*} u^{\prime} & \text { en } & L^{\infty}\left(0, T ; H_{0}^{1}(\Omega) \cap H^{2}(\Omega)\right), \\
u_{\nu}^{\prime} \stackrel{*}{\rightarrow} u^{\prime} & \text { en } & L^{\infty}\left(0, T ; L^{2}(\Omega)\right), \\
u_{\nu}^{\prime \prime} \stackrel{*}{\rightarrow} u^{\prime \prime} & \text { en } & L^{\infty}\left(0, T ; L^{2}(\Omega)\right) .
\end{array}
$$

Por pasaje al límite en la ecuación (3.3) y utilizando (3.19), resulta

$$
\int_{0}^{t} \int_{\Omega}\left[u^{\prime \prime}+\beta(x) u^{\prime}+\alpha \Delta^{2} u-\mu(t) \Delta u-h(x, t)\right] v \theta d x d t=0,
$$


para todo $\theta \in D(0, T)$ y para todo $v \in U$. Por esta identidad, tenemos

$$
u^{\prime \prime}+\beta(x) u^{\prime}+\alpha \Delta^{2} u-\mu(t) \Delta u=h(x, t) \text { en } D^{\prime}(\Omega \times] 0, T[) .
$$

Por otra parte, desde que $u^{\prime \prime}, \beta(x) u^{\prime}, \mu(t) \Delta u$ y $h(x, t)$ pertenecen al espacio $L^{\infty}\left(0, T ; L^{2}(\Omega)\right)$ y por (3.20), se deduce que $\Delta^{2} u \in L^{\infty}\left(0, T ; L^{2}(\Omega)\right)$. En consecuencia $u \in U$ y se tiene

$$
u^{\prime \prime}+\beta(x) u^{\prime}+\alpha \Delta^{2} u-\mu(t) \Delta u=h(x, t) \text { en } L^{\infty}\left(0, T ; L^{2}(\Omega)\right) .
$$

Los datos iniciales se verifican de modo estándar. La estimativa (3.2) se obtiene de manera similar que (3.8). La unicidad resulta de la estimativa (3.2). Esto concluye la demostración del Lema 3.1.

Lema 3.2. Supongamos que las funciones $\mu$ y $\beta$ satisfacen las hipótesis (H1) y (H2) respectivamente, $u_{0} \in H_{0}^{2}, u_{1} \in L^{2}(\Omega)$ y $h \in W^{1,2}\left(0, T ; L^{2}(\Omega)\right)$. Entonces el problema (3.1) admite solución única $u$ sobre $[0, T]$ tal que

$$
\begin{aligned}
& u \in C\left([0, T] ; H_{0}^{2}(\Omega)\right), \\
& u^{\prime} \in C\left([0, T] ; L^{2}(\Omega)\right), \\
& u^{\prime \prime} \in L^{\infty}\left(0, T ; H^{-1}(\Omega)\right) .
\end{aligned}
$$

Además, la solución u verifica la estimativa (3.2).

Demostración. Como $U$ es denso en $H_{0}^{2}(\Omega)$ y $H_{0}^{1}(\Omega) \cap H^{2}(\Omega)$ es denso en $L^{2}(\Omega)$, entonces existen sucesiones $\left\{u_{0 m}\right\} \subset U$ y $\left\{u_{1 m}\right\} \subset H_{0}^{1}(\Omega) \cap H^{2}(\Omega)$ tales que

$$
\begin{aligned}
& u_{0 m} \longrightarrow u_{0} \text { en } H_{0}^{2}(\Omega), \\
& u_{1 m} \longrightarrow u_{1} \text { en } L^{2}(\Omega) .
\end{aligned}
$$

Ahora consideremos para cada $m \in \mathbb{N}$, el siguiente problema lineal

$$
\begin{array}{ll}
u_{m}^{\prime \prime}+\beta(x) u_{m}^{\prime}+\alpha \Delta^{2} u_{m}-\mu(t) \Delta u_{m}=h(x, t) & \text { en } \Omega \times] 0, T[ \\
u_{m}(x, 0)=u_{0 m}(x), u_{m}^{\prime}(x, 0)=u_{1 m}(x) & \text { en } \Omega, \\
u_{m}=\frac{\partial u_{m}}{\partial \nu}=0 & \text { en } \partial \Omega \times] 0, T[.
\end{array}
$$

Por el Lema 3.1, para cada $m \in \mathbb{N}$, existe una única función $u_{m}$ tal que

$$
\begin{gathered}
u_{m}^{\prime \prime}+\beta(x) u_{m}^{\prime}+\alpha \Delta^{2} u_{m}-\mu(t) \Delta u_{m}=h(x, t) \text { en } L^{\infty}\left(0, T ; L^{2}(\Omega)\right) \\
u_{m} \in L^{\infty}(0, T ; U) \\
u_{m}^{\prime} \in L^{\infty}\left(0, T ; H_{0}^{1}(\Omega) \cap H^{2}(\Omega)\right) \\
u_{m}^{\prime \prime} \in L^{\infty}\left(0, T ; L^{2}(\Omega)\right)
\end{gathered}
$$

y verifica la estimativa (3.2).

De las pertenencias (3.25), obtenemos

$$
\begin{aligned}
& u_{m} \in C\left([0, T] ; H_{0}^{2}(\Omega)\right), \\
& u_{m}^{\prime} \in C\left([0, T] ; L^{2}(\Omega)\right) .
\end{aligned}
$$

Por (3.22), $\left\{u_{0 m}\right\}$ y $\left\{u_{1 m}\right\}$ son sucesiones de Cauchy en $H_{0}^{2}(\Omega)$ y en $L^{2}(\Omega)$ respectivamente. Por la estimativa (3.2), resulta que $\left\{u_{m}\right\}$ y $\left\{u_{m}^{\prime}\right\}$ son sucesiones de Cauchy en $C\left([0, T] ; H_{0}^{2}(\Omega)\right)$ y en $C\left([0, T] ; L^{2}(\Omega)\right)$ respectivamente. Así existen $u \in C\left([0, T] ; H_{0}^{2}(\Omega)\right)$ y $u^{\prime} \in C\left([0, T] ; L^{2}(\Omega)\right)$ tales que

$$
\begin{array}{ll}
u_{m} \longrightarrow u & \text { en } \quad C\left([0, T] ; H_{0}^{2}(\Omega)\right), \\
u_{m}^{\prime} \longrightarrow u^{\prime} & \text { en } \quad C\left([0, T] ; L^{2}(\Omega)\right) .
\end{array}
$$

Por (3.26) y de (3.24), por pasaje al límite, se obtiene (3.20). Desde que $u \in C\left([0, T] ; H_{0}^{2}(\Omega)\right)$, se obtienen $\Delta u \in L^{\infty}\left(0, T ; L^{2}(\Omega)\right)$ y $\Delta^{2} u \in L^{\infty}\left(0, T ; H^{-1}(\Omega)\right)$. De $(3.20)$ resulta $u^{\prime \prime} \in L^{\infty}\left(0, T ; H^{-1}(\Omega)\right)$ y se tiene

$$
u^{\prime \prime}+\beta(x) u^{\prime}+\alpha \Delta^{2} u-\mu(t) \Delta u=h(x, t) \text { en } L^{\infty}\left(0, T ; H^{-1}(\Omega)\right) .
$$

De aquí el resto es similar al Lema 3.1. Esto concluye la demostración del Lema 3.2. 


\section{EXISTENCIA LOCAL}

En esta sección, discutiremos la existencia y la unicidad de la solución local del problema (1.1) - (1.3), usando argumentos del teorema de punto fijo de Banach. Para esto necesitaremos los resultados del Lema 3.2 .

Definamos el siguiente espacio de dos parámetros, llamado Conjunto de Soluciones o Conjunto Admisible

$$
\begin{aligned}
& G_{T_{0}, R_{0}}:=\left\{v \in C\left(\left[0, T_{0}\right] ; H_{0}^{2}(\Omega)\right) ; v^{\prime} \in C\left(\left[0, T_{0}\right] ; L^{2}(\Omega)\right),\right. \\
& \left|u^{\prime}(t)\right|_{2}^{2}+|\Delta u(t)|_{2}^{2} \leq R_{0}^{2}, t \in\left[0, T_{0}\right] \\
& \left.\operatorname{con} v(0)=u_{0} \mathrm{y} v^{\prime}(0)=u_{1}\right\} \text {, }
\end{aligned}
$$

donde $T_{0}>0$ y $R_{0}>0$. Entonces $G_{T_{0}, R_{0}}$ es un espacio métrico completo con la distancia

$$
d(u, v):=\sup _{0 \leq t \leq T_{0}}\left[\left|u^{\prime}(t)-v^{\prime}(t)\right|_{2}^{2}+|\Delta u(t)-\Delta v(t)|_{2}^{2}\right]^{\frac{1}{2}}
$$

donde $u, v \in G_{T_{0}, R_{0}}$.

Lema 4.1. Supongamos que la función $M$ satisface la hipótesis (H3). Si $u, v \in G_{T_{0}, R_{0}}$ y $\mu(t ; u):=$ $M\left(\|u(t)\|^{2}\right)$, entonces $\mu(. ; u) \in C\left(\left[0, T_{0}\right]\right)$ y $\mu^{\prime}(. ; u) \in L^{\infty}\left(0, T_{0}\right)$. Además se verifica para cada $t, s \in\left[0, T_{0}\right]$ las siguientes desigualdades:

$$
\begin{gathered}
|\mu(t ; u)-\mu(s ; u)| \leq 2 M_{1} R_{0}^{2}|t-s|, \\
|\mu(t ; u)-\mu(t ; v)| \leq 2 M_{1} B_{1}^{2} R_{0}|\Delta u(t)-\Delta v(t)|_{2}, \\
\left|\mu^{\prime}(t ; u)\right| \leq 2 M_{1} R_{0}^{2} \\
0<m_{0} \leq \mu(t ; u) \leq M_{0},
\end{gathered}
$$

donde $M_{0}:=\sup \left\{M(s) ; 0 \leq s \leq B_{1}^{2} R_{0}^{2}\right\}, B_{1}$ es la constante de la desigualdad de Sobolev-Poincaré y $M_{1}:=\sup \left\{\left|M^{\prime}(s)\right| ; 0 \leq s \leq B_{1}^{2} R_{0}^{2}\right\}$.

Demostración. Por el teorema de Valor Medio, se obtiene

$$
|\mu(t ; u)-\mu(s ; u)|=\left|2 M^{\prime}\left(\|u(\xi)\|^{2}\right)\left(-\Delta u(\xi), u^{\prime}(\xi)\right)\right||t-s|
$$

y

$$
\begin{aligned}
|\mu(t ; u)-\mu(t ; v)| & =\left|M^{\prime}(\eta)\right|(\|u(t)\|+\|v(t)\|)|\|u(t)\|-\|v(t)\|| \\
& \leq\left|M^{\prime}(\eta)\right|(\|u(t)\|+\|v(t)\|)\|u(t)-v(t)\|,
\end{aligned}
$$

donde $\xi$ está entre $t$ y $s, \eta$ está entre $\|u(t)\|^{2}$ y $\|v(t)\|^{2}$. Aplicando la desigualdad de Sobolev-Poincaré, a las relaciones anteriores se obtienen los resultados.

Lema 4.2. Supongamos que la función $f$ satisface la hipótesis (H4). Si $u, v \in G_{T_{0}, R_{0}}$ y $h(t ; v):=$ $f(v(t))$, entonces $h \in C\left(\left[0, T_{0}\right] ; L^{2}(\Omega)\right)$. Además se verifica para cada $t, s \in\left[0, T_{0}\right]$ las siguientes desigualdades:

$$
\begin{gathered}
|h(t ; v)-h(s ; v)|_{2} \leq 2 K B_{1}^{2(p-1)} R_{0}^{p-2}|\Delta v(t)-\Delta v(s)|_{2}, \\
|h(t ; u)-h(t ; v)|_{2} \leq 2 K B_{1}^{2(p-1)} R_{0}^{p-2}|\Delta u(t)-\Delta v(t)|_{2}, \\
|h(t ; v)|_{2} \leq K B_{1}^{2(p-1)} R_{0}^{p-1}
\end{gathered}
$$

donde $B_{1}$ es la constante de la desigualdad de Sobolev-Poincaré. 
Demostración. Por la hipótesis $(H 4)$, resulta

$$
\begin{aligned}
|h(t ; v)-h(s ; v)|_{2} & =|f(v(t))-f(v(s))|_{2} \\
& \leq K|| v(t)-v(s)\left|\left(|v(t)|^{p-2}+|v(s)|^{p-2}\right)\right|_{2} \\
& \leq K|v(t)-v(s)|_{q}\left(|v(t)|_{r(p-2)}^{p-2}+|v(s)|_{r(p-2)}^{p-2}\right)
\end{aligned}
$$

donde $\frac{1}{r}+\frac{1}{q}=\frac{1}{2}$. Aplicando la desigualdad de Sobolev-Poincaré, se obtiene

$$
\begin{aligned}
|h(t ; v)-h(s ; v)|_{2} & \leq K B_{1}^{2(p-1)}|\Delta v(t)-\Delta v(s)|_{2}\left(|\Delta v(t)|_{2}^{p-2}+|\Delta v(s)|_{2}^{p-2}\right) \\
& \leq 2 K B_{1}^{2(p-1)} R_{0}^{p-2}|\Delta u(t)-\Delta u(s)|_{2} .
\end{aligned}
$$

Recurriendo nuevamente a la hipotesis $(H 4)$ y la desigualdad de Sobolev-Poincaré, se obtiene

$$
\begin{aligned}
|h(t ; v)|_{2} & =|f(v(t))|_{2} \\
& \leq K|v(t)|_{2(p-1)}^{p-1} \\
& \leq K B_{1}^{2(p-1)}|\Delta v(t)|_{2}^{p-1} \\
& \leq K B_{1}^{2(p-1)} R_{0}^{p-1} .
\end{aligned}
$$

Observar, que en la aplicación de la desigualdad de Sobolev-Poincaré, se ha utilizado la condición de $p$. Esto concluye la demostración.

Teorema 4.3 (Existencia Local). Supongamos que las funciones $\beta, M$ y $f$ satisfacen las hipótesis (H2), (H3) y (H4) respectivamente, $u_{0} \in H_{0}^{2}(\Omega)$ y $u_{1} \in L^{2}(\Omega)$. Entonces existe $T_{0}>0$, de tal modo que el problema $(1.1)-(1.3)$ admite solución única u sobre $\left[0, T_{0}\right]$ tal que

$$
\begin{aligned}
& u \in C\left(\left[0, T_{0}\right] ; H_{0}^{2}(\Omega)\right), \\
& u^{\prime} \in C\left(\left[0, T_{0}\right] ; L^{2}(\Omega)\right), \\
& u^{\prime \prime} \in L^{\infty}\left(0, T_{0} ; H^{-1}(\Omega)\right) .
\end{aligned}
$$

Demostración. Procedemos en dos etapas.

Existencia de Soluciones. Para cada $v \in G_{T_{0}, R_{0}}$, consideremos la siguiente ecuación lineal

$$
\left.u^{\prime \prime}+\beta(x) u^{\prime}+\alpha \Delta^{2} u-\mu(t ; v) \Delta u=h(t ; v) \text { en } \Omega \times\right] 0, T_{0}[,
$$

con condiciones iniciales

$$
u(x, 0)=u_{0}(x), u^{\prime}(x, 0)=u_{1}(x), \text { en } \Omega
$$

y condiciones de frontera

$$
\left.u=\frac{\partial u}{\partial \nu}=0, \text { en } \partial \Omega \times\right] 0, T_{0}[,
$$

donde $T_{0}>0$ y $R_{0}>0$ serán obtenidos posteriormente, $h(t ;, v):=f(v(t))$ y $\mu(t ; v):=M\left(\|v(t)\|^{2}\right)$. Por los Lemas 4.1 y 4.2 , las funciones $\mu(. ; v)$ y $h(. ; v)$, satisfacen las hipótesis del Lema 3.2, entonces existe solución única $u$ sobre $\left[0, T_{0}\right]$ del problema $(4.2)-(4.4)$ tal que

$$
\begin{aligned}
& u \in C\left([0, T] ; H_{0}^{2}(\Omega)\right), \\
& u^{\prime} \in C\left([0, T] ; L^{2}(\Omega)\right), \\
& u^{\prime \prime} \in L^{\infty}\left(0, T ; H^{-1}(\Omega)\right) .
\end{aligned}
$$

Además, la solución $u$ verifica para cada $t \in\left[0, T_{0}\right]$, la siguiente estimativa

$$
E_{1}(t) \leq\left[E_{1}^{\frac{1}{2}}(0)+\int_{0}^{t}|h(s ; v)|_{2} d s\right]^{2} \exp \left(\int_{0}^{t}\left[\frac{\left|\mu^{\prime}(s ; v)\right|}{\mu(s ; v)}+|\beta|_{L^{\infty}}\right] d s\right)
$$


donde

$$
\begin{aligned}
& E_{1}(t):=\frac{1}{2}\left|u^{\prime}(t)\right|_{2}^{2}+\frac{1}{2} \alpha|\Delta u(t)|_{2}^{2}+\frac{1}{2} \mu(t ; v)\|u(t)\|^{2}, \\
& E_{1}(0):=\frac{1}{2}\left|u_{1}\right|_{2}^{2}+\frac{1}{2} \alpha\left|\Delta u_{0}\right|_{2}^{2}+\frac{1}{2} M\left(\left\|u_{0}\right\|^{2}\right)\left\|u_{0}\right\|^{2} .
\end{aligned}
$$

El siguiente paso será mostrar que $u \in G_{T_{0}, R_{0}}$. Por (4.6) y los Lemas 4.1 y 4.2 , se obtiene

$$
\begin{aligned}
\left|u^{\prime}(t)\right|_{2}^{2}+|\Delta u(t)|_{2}^{2} \leq & \frac{2}{\min \{1, \alpha\}}\left[E_{1}^{\frac{1}{2}}(0)+K B_{1}^{2(p-1)} R_{0}^{p-1} T_{0}\right]^{2} \\
& \exp \left(\int_{0}^{T_{0}}\left[\frac{2 M_{1} R_{0}^{2}}{m_{0}}+|\beta|_{L^{\infty}}\right] d s\right) \cdot 4.7
\end{aligned}
$$

Escogemos la constante $R_{0}>0$ que satisfaga la relación

$$
R_{0} \geq\left(\frac{4}{\operatorname{mín}\{1, \alpha\}}\right)^{\frac{1}{2}}\left[E_{1}^{\frac{1}{2}}(0)+1\right]
$$

y consideremos $T_{0}>0$ que satisfaga las relaciones

$$
K B_{1}^{2(p-1)} R_{0}^{p-1} T_{0} \leq 1 \text { y } \exp \left(\left[\frac{2 M_{1} R_{0}^{2}}{m_{0}}+|\beta|_{L^{\infty}}\right] T_{0}\right) \leq 2 .
$$

Entonces de (4.7) utilizando (4.8) y (4.9), resulta

$$
\left|u^{\prime}(t)\right|_{2}^{2}+|\Delta u(t)|_{2}^{2} \leq R_{0}^{2}, \forall t \in\left[0, T_{0}\right] .
$$

Por (4.5), (4.10) y como $u$ es solución de (4.2) - (4.4), se tiene que $u \in G_{T_{0}, R_{0}}$.

Por el resultado antes obtenido, tiene sentido definir la aplicación no lineal $\varphi$ como sigue:

$$
\begin{aligned}
\varphi: G_{T_{0}, R_{0}} & \longrightarrow G_{T_{0}, R_{0}} \\
v & \longmapsto \varphi(v)=u
\end{aligned}
$$

donde $u$ es la solución del problema (4.2) - (4.4) para $v \in G_{T_{0}, R_{0}}$. Esto significa que $\varphi\left(G_{T_{0}, R_{0}}\right) \subset G_{T_{0}, R_{0}}$.

Ahora probaremos que $\varphi$ es una contracción estricta con respecto a la distancia (4.1), es decir, se cumple para cada $v_{1}, v_{2} \in G_{T_{0}, R_{0}}$ la desigualdad

$$
d\left(\varphi\left(v_{1}\right), \varphi\left(v_{2}\right)\right) \leq \delta d\left(v_{1}, v_{2}\right),
$$

para un fijo $\delta, 0<\delta<1$.

Sean $v_{1}, v_{2} \in G_{T_{0}, R_{0}}$, entonces $u_{1}=\varphi\left(v_{1}\right)$ y $u_{2}=\varphi\left(v_{2}\right)$ son soluciones del problema (4.2) - (4.4). Haciendo, $Q:=\Omega \times] 0, T_{0}[, \Sigma:=\partial \Omega \times] 0, T_{0}\left[, w=u_{1}-u_{2}\right.$, entonces $w$ satisface el problema lineal

$$
\begin{array}{ll}
w^{\prime \prime}+\beta(x) w^{\prime}+\alpha \Delta^{2} w-\mu\left(t ; v_{1}\right) \Delta w=h\left(t ; v_{1}, v_{2}\right) & \text { en } Q \\
w(x, 0)=0, w^{\prime}(x, 0)=0 & \text { en } \Omega, \\
w=\frac{\partial w}{\partial \nu}=0 & \text { en } \Sigma
\end{array}
$$

donde

$$
\begin{aligned}
& \mu(t ; v):=M\left(\|v(t)\|^{2}\right) \\
& h\left(t ; v_{1}, v_{2}\right):=\left[\mu\left(t ; v_{1}\right)-\mu\left(t ; v_{2}\right)\right] \Delta u_{2}(t)+f\left(v_{1}\right)-f\left(v_{2}\right) .
\end{aligned}
$$

Por los Lemas 4.1 y 4.2 , obtenemos

$$
\left|h\left(t ; v_{1}, v_{2}\right)\right|_{2} \leq 2\left[M_{1} B_{1}^{2} R_{0}^{2}+K B_{1}^{2(p-1)} R_{0}^{p-2}\right]\left|\Delta v_{1}(t)-\Delta v_{2}(t)\right|_{2} .
$$


Del Lema 4.1, (4.12) y Lema 3.2, existe una única solución $w$ sobre $\left[0, T_{0}\right]$ del problema (4.11) y se verifica para cada $t \in\left[0, T_{0}\right]$ la estimativa

$$
E_{1}(t) \leq\left[\int_{0}^{t}\left|h\left(s ; v_{1}, v_{2}\right)\right|_{2} d s\right]^{2} \exp \left(\int_{0}^{t}\left[\frac{\left|\mu^{\prime}\left(s ; v_{1}\right)\right|}{\mu\left(s ; v_{1}\right)}+|\beta|_{L^{\infty}}\right] d s\right)
$$

donde

$$
E_{1}(t):=\frac{1}{2}\left|w^{\prime}(t)\right|_{2}^{2}+\frac{1}{2} \alpha|\Delta w(t)|_{2}^{2}+\frac{1}{2} \mu\left(t ; v_{1}\right)\|w(t)\|^{2} .
$$

Utilizando el Lema 4.1, (4.9) y (4.12) en (4.13), se obtiene

$$
\begin{aligned}
{\left[\left|w^{\prime}(t)\right|_{2}^{2}+|\Delta w(t)|_{2}^{2}\right]^{\frac{1}{2} \leq} } & \left(\frac{16}{\min \{1, \alpha\}}\right)^{\frac{1}{2}}\left[M_{1} B_{1}^{2} R_{0}^{2}+K B_{1}^{2(p-1)} R_{0}^{p-2}\right] \\
& \int_{0}^{t}\left|\Delta v_{1}(s)-\Delta v_{2}(s)\right|_{2} d s .4 .14
\end{aligned}
$$

Aplicando supremo en (4.14) y utilizando (4.9), resulta

$$
d\left(\varphi\left(v_{1}\right), \varphi\left(v_{2}\right)\right) \leq\left(\frac{16}{\operatorname{mí}\{1, \alpha\}}\right)^{\frac{1}{2}}\left[M_{1} B_{1}^{2} R_{0}^{2} T_{0}+R_{0}^{-1}\right] d\left(v_{1}, v_{2}\right) .
$$

Además de las relaciones de (4.9), y tomando un $T_{0}>0$ que satisfaga la relación

$$
\delta:=\left(\frac{16}{\min \{1, \alpha\}}\right)^{\frac{1}{2}}\left[M_{1} B_{1}^{2} R_{0}^{2} T_{0}+R_{0}^{-1}\right]<1,
$$

se tiene de (4.15) que $\varphi$ es una contracción estricta.

Aplicando el teorema de Punto Fijo de Banach, existe un único $u \in G_{T_{0}, R_{0}}$ tal que $\varphi(u)=u$. Así, hemos obtenido la existencia de la solución local del problema (1.1) - (1.3)

Unicidad de Soluciones. Sea $u$ la solución obtenida en la prueba de existencia, la cual pertenece a $G_{T_{0}, R_{0}}$. Consideremos otra solución $v$ del problema (1.1) - (1.3) tal que

$$
\begin{aligned}
& v \in C\left(\left[0, T_{1}\right] ; H_{0}^{2}(\Omega)\right), \\
& v^{\prime} \in C\left(\left[0, T_{1}\right] ; L^{2}(\Omega)\right), \\
& v^{\prime \prime} \in L^{\infty}\left(0, T_{1} ; L^{2}(\Omega)\right),
\end{aligned}
$$

con $0<T_{1} \leq T_{0}$. Haciendo, $\left.Q:=\Omega \times\right] 0, T_{1}[, \Sigma:=\partial \Omega \times] 0, T_{1}[$ y $w=u-v$, entonces la función $w$ satisface el problema lineal

$$
\begin{array}{ll}
w^{\prime \prime}+\beta(x) w^{\prime}+\alpha \Delta^{2} w-\mu(t ; u) \Delta w=h(t ; u, v) & \text { en } Q, \\
w(x, 0)=0, w^{\prime}(x, 0)=0 & \text { en } \Omega, \\
w=\frac{\partial w}{\partial \nu}=0 & \text { en } \Sigma,
\end{array}
$$

donde

$$
\begin{aligned}
& \mu(t ; z):=M\left(\|z(t)\|^{2}\right), \\
& h(t ; u, v):=[\mu(t ; u)-\mu(t ; v)] \Delta v(t)+f(u(t))-f(v(t)) .
\end{aligned}
$$

Por (4.16), existe una constante $C_{0}>0$ tal que

$$
|\Delta v(t)|_{2} \leq C_{0}, \forall t \in\left[0, T_{1}\right] .
$$

Por el teorema de Valor Medio, desigualdad de Sobolev-Poincaré y (4.18), se obtiene

$$
\begin{aligned}
|h(t ; u, v)|_{2} \leq & {\left[M_{2} C_{0} B_{1}^{2}\left(R_{0}+C_{0}\right)\right.} \\
& \left.+K B_{1}^{2(p-1)}\left(R_{0}^{p-2}+C_{0}^{p-2}\right)\right]|\Delta w(t)|_{2} \\
= & K_{0}|\Delta w(t)|_{2} 4.19
\end{aligned}
$$


donde $M_{2}:=\sup \left\{\left|M^{\prime}(s)\right| ; 0 \leq s \leq B_{1}^{2} \operatorname{máx}\left\{R_{0}^{2}, C_{0}^{2}\right\}\right\}$ y $K_{0}$ es una constante positiva.

Del Lema $4.1,(4.19)$ y Lema 3.2 , existe una única solución $w$ sobre $\left[0, T_{1}\right]$ del problema (4.17) y verifica la estimativa

$$
E_{1}(t) \leq\left[\int_{0}^{t}|h(s ; u, v)|_{2} d s\right]^{2} \exp \left(\int_{0}^{t}\left[\frac{\left|\mu^{\prime}(s ; u)\right|}{\mu(s ; u)}+|\beta|_{L^{\infty}}\right] d s\right)
$$

donde

$$
E_{1}(t):=\frac{1}{2}\left|w^{\prime}(t)\right|_{2}^{2}+\frac{1}{2} \alpha|\Delta w(t)|_{2}^{2}+\frac{1}{2} \mu(t ; u)\|w(t)\|^{2} .
$$

De (4.20) y empleando el Lema 4.1 y (4.19), resulta

$$
|\Delta w(t)|_{2} \leq C_{4} \int_{0}^{t}|\Delta w(s)|_{2} d s, \forall t \in\left[0, T_{1}\right]
$$

donde $C_{4}$ es una constante positiva.

De (4.21) y la desigualdad de Gronwall, resulta $|\Delta w(t)|_{2}=0, \forall t \in\left[0 . T_{1}\right], 0<T_{1} \leq T_{0}$. Esto implica que $u(t)=v(t)=0, \forall t \in\left[0, T_{1}\right]$. Con esto hemos probado la unicidad de la solución y por tanto finalizado la demostración del Teorema 4.3.

Corolario 4.4. Supongamos que las funciones $\beta, M$ y $f$ satisfacen las hipótesis (H2), (H3) y (H4) respectivamente, $u_{0} \in H_{0}^{2}(\Omega)$ y $u_{1} \in L^{2}(\Omega)$. Entonces existe un único intervalo $\left[0, T_{\operatorname{máx}}[\right.$ con $0<T_{\operatorname{máx}} \leq \infty$ y el problema $(1.1)-(1.3)$ admite solución única u sobre $\left[0, T_{\operatorname{máx}}[\right.$ tal que

$$
\begin{aligned}
& u \in C\left(\left[0, T_{\text {máx }}\left[; H_{0}^{2}(\Omega)\right),\right.\right. \\
& u^{\prime} \in C\left(\left[0, T_{\operatorname{máx}}\left[; L^{2}(\Omega)\right),\right.\right. \\
& u^{\prime \prime} \in L^{\infty}\left(0, T_{\text {máx }} ; L^{2}(\Omega)\right) .
\end{aligned}
$$

Demostración. Similar a la demostración del Corolario 4.4 [10].

Observación 4.5. Si consideramos en el problema (1.1) - (1.3) que el coeficiente $\beta(x, t)$ del término disipativo es una función real para $x \in \Omega, t \geq 0$ tal que $\beta \in W^{2,2}\left(0, \infty ; L^{\infty}(\Omega)\right)$, entonces se obtienen los mismo resultados de los Lemas 3.1 y 3.2, Teorema 4.3 y Corolario 4.4. La hipótesis (H2) para $\beta$ es solamente necesaria para obtener la propiedad de singularidad.

\section{PROPIEDAD DE SINGULARIDAD}

En esta sección, discutiremos la propiedad de singularidad en tiempo finito de la solución del problema (1.1) - (1.3) sobre un intervalo maximal $\left[0, T_{\text {máx }}[\right.$. En la discusión usaremos el método directo $[5,13]$.

Definición 5.1. Una solución $u$ del problema $(1.1)$ - (1.3) sobre $\left[0, T_{\text {máx }}\right.$ [ tiene la propiedad de explosión o singularidad en tiempo finito, si

$$
T_{\text {máx }}<\infty \text { y } \lim _{t \rightarrow T_{\text {máx }}^{-}} \int_{\Omega}|u(x, t)|^{2} d x=\infty .
$$

Definición 5.2. La función energía $E(t)$ del problema (1.1) - (1.3), se define por

$$
E(t):=\frac{1}{2}\left|u^{\prime}(t)\right|_{2}^{2}+\frac{1}{2} \alpha|\Delta u(t)|_{2}^{2}+\frac{1}{2} \widehat{M}\left(\|u(t)\|^{2}\right)-\int_{\Omega} F(u(x, t)) d x,
$$

para $t \geq 0$, donde

$$
\widehat{M}(s):=\int_{0}^{s} M(\xi) d \xi \text { y } F(s):=\int_{0}^{s} f(\xi) d \xi
$$


Lema 5.3. Supongamos que se cumplen las hipótesis $(H 2)-(H 4)$. Si u es una solución del problema (1.1) - (1.3) sobre $\left[0, T_{\text {máx }}\left[\right.\right.$ con datos iniciales $u_{0} \in H_{0}^{2}(\Omega)$ y $u_{1} \in L^{2}(\Omega)$, entonces

$$
E(t)+\int_{0}^{t}\left|\sqrt{\beta} u^{\prime}(s)\right|_{2}^{2} d s=E(0),
$$

para $t \geq 0$, donde $E(0)$ es la energía inicial definida por

$$
E(0):=\frac{1}{2}\left|u_{1}\right|_{2}^{2}++\frac{1}{2} \alpha\left|\Delta u_{0}\right|_{2}^{2} \frac{1}{2} \widehat{M}\left(\left\|u_{0}\right\|^{2}\right)-\int_{\Omega} F\left(u_{0}(x)\right) d x .
$$

Demostración. Multiplicando a la ecuación (1.1) por $u_{t}$, integrando sobre $\Omega$ y utilizando el teorema de la Divergencia, se obtiene

$$
E^{\prime}(t)+\left|\sqrt{\beta} u^{\prime}(t)\right|_{2}^{2}=0
$$

De aquí, se tiene el resultado.

Lema 5.4. Sea la función definida para $\lambda \geq 0$ :

$$
g(\lambda):=\frac{1}{2} \delta \lambda^{2}-\frac{K B_{1}^{p}}{p} \lambda^{p},
$$

donde $\delta:=\frac{\alpha}{B_{1}^{2}}+m_{0}, B_{1}$ es la constante de la desigualdad de Sobolev-Poincaré; $m_{0}, K$ y $p$ son constantes de las hipótesis $(H 3)-(H 4)$. Entonces

(i) $g$ es estrictamente creciente en $\left[0, \lambda_{0}[\right.$,

(ii) g toma su valor máximo $E_{0}$ en $\lambda_{0}$,

(iii) $g$ es estrictamente decreciente en $] \lambda_{0}, \infty[$,

donde

$$
\lambda_{0}:=\left(\frac{\delta}{K B_{1}^{p}}\right)^{\frac{1}{p-2}} \quad y \quad E_{0}:=\left(\frac{1}{2}-\frac{1}{p}\right) \delta^{\frac{p}{p-2}}\left(K B_{1}^{p}\right)^{\frac{-2}{p-2}}
$$

Demostración. La verificación es inmediata.

Lema 5.5. Supongamos que se cumplen las hipótesis $(H 2)-(H 4)$. Si u es una solución del problema (1.1) - (1.3) sobre $\left[0, T_{\operatorname{máx}}\left[\right.\right.$ con datos iniciales $u_{0} \in H_{0}^{2}(\Omega)$ y $u_{1} \in L^{2}(\Omega)$, y que satisface

$$
\left\|u_{0}\right\|>\lambda_{0} \quad \text { y } E(0)<E_{0},
$$

entonces

$$
\|u(t)\|>\lambda_{0}, \text { para } t \geq 0 .
$$

Demostración. Por (5.2), $E(t)$ es una función no creciente y $E(t) \leq E(0)$, para $t \geq 0$. Por la desigualdad de Sobolev-Poincaré y $(H 3)$, resulta

$$
\begin{aligned}
E(t) & \geq \frac{1}{2} \delta\|u(t)\|^{2}-\frac{K B_{1}^{p}}{p}\|u(t)\|^{p} \\
& =g(\|u(t)\|), \quad \text { para } t \geq 0,
\end{aligned}
$$

donde $g$ es la función definida en (5.3). Así se tiene

$$
g(\|u(t)\|) \leq E(t) \leq E(0)<g\left(\lambda_{0}\right):=E_{0}, \text { para } t \geq 0 .
$$

Por (5.6), existe $\lambda_{1}>\lambda_{0}$ tal que $g\left(\lambda_{1}\right)=E(0)$. A continuación probemos que

$$
\|u(t)\| \geq \lambda_{1}, \text { para } t \geq 0 .
$$


Por el absurdo. Supongamos que existe $t_{0}>0$ tal que $\lambda_{0}<\left\|u\left(t_{0}\right)\right\|<\lambda_{1}$. Desde que $g$ es estrictamente decreciente en $] \lambda_{0}, \infty[$, resulta

$$
E(0)=g\left(\lambda_{1}\right)<g\left(\left\|u\left(t_{0}\right)\right\|\right)<g\left(\lambda_{0}\right)
$$

y esto es una contradicción con (5.6). Por tanto se cumple (5.7) y $\|u(t)\|>\lambda_{0}$, para $t \geq 0$. Por tanto, se obtiene (5.5).

Definición 5.6. Para una solución $u$ del problema $(1.1)-(1.3)$ sobre $\left[0, T_{\operatorname{máx}}[\right.$, se define la función explosión

$$
A(t):=|u(t)|_{2}^{2}+\int_{0}^{t}|\sqrt{\beta} u(s)|_{2}^{2} d s, \text { para } t \geq 0
$$

Lema 5.7. Supongamos que se cumplen las hipótesis $(H 2)-(H 6)$. Si u es una solución del problema (1.1) - (1.3) sobre $\left[0, T_{\operatorname{máx}}\left[\right.\right.$ con datos iniciales $u_{0} \in H_{0}^{2}(\Omega)$ y $u_{1} \in L^{2}(\Omega)$, entonces

$$
A^{\prime \prime}(t)-4(\gamma+1)\left[\left|u^{\prime}(t)\right|_{2}^{2}+\int_{0}^{t}\left|\sqrt{\beta} u^{\prime}(s)\right|_{2}^{2} d s\right] \geq Q(t), \text { para } t \geq 0
$$

donde $\gamma$ es la constante dada en $(H 5)$,

$$
Q(t):=-4(2 \gamma+1) E(0)+4 \gamma \delta\|u(t)\|^{2}
$$

y $\delta$ es la constante de la función (5.3).

Demostración. Por diferenciación de (5.8), se tiene

$$
A^{\prime}(t)=2\left(u^{\prime}(t), u(t)\right)+|\sqrt{\beta} u(t)|_{2}^{2} .
$$

Diferenciando (5.11), utilizando la ecuación (1.1) y el teorema de la Divergencia, se obtiene

$$
\begin{aligned}
A^{\prime \prime}(t)= & 2\left|u^{\prime}(t)\right|_{2}^{2}-2 \alpha|\Delta u(t)|_{2}^{2}-2 M\left(\|u(t)\|^{2}\right)\|u(t)\|^{2} \\
& +2(f(u(t)), u(t)) .5 .12
\end{aligned}
$$

Por (5.1), se obtiene de (5.12)

$$
\begin{aligned}
A^{\prime \prime}(t)-4 & (\gamma+1)\left[\left|u^{\prime}(t)\right|_{2}^{2}+\int_{0}^{t}\left|\sqrt{\beta} u^{\prime}(s)\right|_{2}^{2} d s\right] \\
= & -4(2 \gamma+1) E(0)+4 \gamma \alpha|\Delta u(t)|_{2}^{2} \\
& +2 \int_{\Omega}[f(u) u-2(2 \gamma+1) F(u)] d x \\
& +2\left[(2 \gamma+1) \widehat{M}\left(\|u(t)\|^{2}\right)-M\left(\|u(t)\|^{2}\right)\|u(t)\|^{2}\right] \\
& +4 \gamma \int_{0}^{t}\left|\sqrt{\beta} u^{\prime}(s)\right|_{2}^{2} d s .
\end{aligned}
$$

Por las hipótesis $(H 2)-(H 6)$ y utilizando la desigualdad de Sobolev-Poincaré, se obtiene de (5.13) el resultado (5.9).

Lema 5.8. Supongamos que se cumplen las hipótesis $(\mathrm{H2})-(\mathrm{H6})$. Si u es una solución del problema (1.1) - (1.3) sobre $\left[0, T_{\text {máx }}\left[\right.\right.$ con datos iniciales $u_{0} \in H_{0}^{2}(\Omega)$ y $u_{1} \in L^{2}(\Omega)$, y satisfaciendo una de las siguientes condiciones:

(i) $E(0)<0$,

(ii) $E(0)=0$ y $\quad A^{\prime}(0)>K_{0}$,

(iii) $0<E(0)<E_{0} \quad y \quad\left\|u_{0}\right\|>\lambda_{0}$, 
(iv) $E(0) \geq E_{0} \quad y \quad A^{\prime}(0)>r_{2}\left[A(0)+\frac{K_{1}}{4(\gamma+1)}\right]+K_{0}$,

donde $\lambda_{0}$ y $E_{0}$ son constantes dadas en (5.4),

$$
\begin{gathered}
K_{0}:=\left|\sqrt{\beta} u_{0}\right|_{2}^{2} \\
A(0):=\left|u_{0}\right|_{2}^{2}, \quad A^{\prime}(0):=2\left(u_{1}, u_{0}\right)+K_{0} \\
K_{1}:=4(2 \gamma+1) E(0)+4(\gamma+1) K_{0} \\
r_{2}:=2(\gamma+1)-2 \sqrt{(\gamma+1) \gamma}
\end{gathered}
$$

entonces

$$
A^{\prime}(t)>K_{0}, \text { para } t>t_{0}
$$

donde $t_{0}:=\operatorname{máx}\left\{\frac{A^{\prime}(0)-K_{0}}{4(1+2 \gamma) E(0)}, 0\right\}$ en el caso $(i), t_{0}:=\operatorname{máx}\left\{\frac{K_{0}-A^{\prime}(0)}{K_{2}}, 0\right\}$ en el caso (iii) con $K_{2}:=$ $2 p\left(E_{0}-E(0)\right)$ y $t_{0}:=0$ en los casos (ii) y (iv).

Demostración. Consideremos cuatro casos de acuerdo al signo de la energía inicial $E(0)$.

(i) $\mathrm{Si} E(0)<0$, de $(5.9)$, se tiene

$$
A^{\prime \prime}(t) \geq-4(2 \gamma+1) E(0)
$$

e integrando, resulta

$$
A^{\prime}(t) \geq A^{\prime}(0)-4(2 \gamma+1) E(0) t \text {, para } t \geq 0 .
$$

Considerando $A^{\prime}(0)-K_{0}-4(2 \gamma+1) E(0) t>0$, se obtiene

$$
A^{\prime}(t)>K_{0}, \text { para } t>t_{0},
$$

donde

$$
t_{0}:=\operatorname{máx}\left\{\frac{A^{\prime}(0)-K_{0}}{4(1+2 \gamma) E(0)}, 0\right\} .
$$

(ii) Si $E(0)=0$, de (5.9), se tiene

$$
A^{\prime \prime}(t) \geq 0
$$

e integrando, resulta

$$
A^{\prime}(t) \geq A^{\prime}(0), \text { para } t \geq 0 .
$$

Considerando $A^{\prime}(0)-K_{0}>0$, se obtiene

$$
A^{\prime}(t) \geq K_{0}, \text { para } t>0 .
$$

(iii) Para $0<E(0)<E_{0}$ y $\left\|u_{0}\right\|>\lambda_{0}$. De (5.3) y Lema 5.5 , vemos que

$$
\begin{aligned}
Q(t) & >-4(2 \gamma+1) E(0)+4 \gamma \delta^{\frac{P}{P-2}}\left(K B_{1}^{p}\right)^{\frac{-2}{p-2}} \\
& =4(2 \gamma+1)\left[-E(0)+\frac{\gamma}{2 \gamma+1} \frac{2 p}{p-2} E_{0}\right] .5 .15
\end{aligned}
$$

Tomando $\gamma=\frac{p-2}{4}$, de (5.9) y (5.15), obtenemos

$$
A^{\prime \prime}(t) \geq Q(t)>K_{2}>0,
$$

donde $K_{2}:=2 p\left(E_{0}-E(0)\right)$. Integrando (5.16), resulta

$$
A^{\prime}(t) \geq A^{\prime}(0)+K_{2} t, \text { para } t \geq 0 .
$$


Considerando $K_{2} t-\left[K_{0}-A^{\prime}(0)\right]>0$, se obtiene

$$
A^{\prime}(t)>K_{0}, \text { para } t>t_{0}
$$

donde

$$
t_{0}:=\operatorname{máx}\left\{\frac{K_{0}-A^{\prime}(0)}{K_{2}}, 0\right\} .
$$

(iv) Para $E(0) \geq E_{0}$. Primero notemos que se cumple

$$
2 \int_{0}^{t}\left(\beta u^{\prime}(s), u(s)\right) d s=|\sqrt{\beta} u(t)|_{2}^{2}-\left|\sqrt{\beta} u_{0}\right|_{2}^{2} .
$$

Usando la desigualdad de Hölder y la desigualdad de Young en (5.17), se obtiene

$$
|\sqrt{\beta} u(t)|_{2}^{2} \leq\left|\sqrt{\beta} u_{0}\right|_{2}^{2}+\int_{0}^{t}|\sqrt{\beta} u(s)|_{2}^{2} d s+\int_{0}^{t}\left|\sqrt{\beta} u^{\prime}(s)\right|_{2}^{2} d s .
$$

Nuevamente usando la desigualdad de Hölder y la desigualdad de Young en (5.11) y por (5.18), resulta

$$
A^{\prime}(t) \leq A(t)+K_{0}+\left|u^{\prime}(t)\right|_{2}^{2}+\int_{0}^{t}\left|\sqrt{\beta} u^{\prime}(s)\right|_{2}^{2} d s
$$

De (5.9) y (5.19), obtenemos

$$
A^{\prime \prime}(t)-4(\gamma+1) A^{\prime}(t)+4(\gamma+1) A(t)+K_{1} \geq 0,
$$

donde

$$
K_{1}:=4(2 \gamma+1) E(0)+4(\gamma+1) K_{0}
$$

Definamos la función

$$
B(t):=A(t)+\frac{K_{1}}{4(\gamma+1)}, \text { para } t \geq 0
$$

Considerando $B^{\prime}(0)>r_{2} B(0)+K_{0}$, la función $B$ satisface las condiciones del Lema 2.3. Así se tiene $A^{\prime}(t)>K_{0}$, para $t>0$. Con esto se concluye la prueba del Lema 5.8.

Definición 5.9. Para las estimativas del tiempo finito de la función explosión $A$, definamos la función

$$
J(t):=\left[A(t)+\left(T_{1}-t\right) K_{0}\right]^{-\gamma}, \text { para } t \in\left[0, T_{1}\right],
$$

donde $T_{1}$ es una constante positiva que será determinada posteriormente y $\gamma$ es la constante dada en (H5).

Teorema 5.10 (Singularidad de Soluciones). Supongamos que se cumplen las hipótesis $(H 2)-(H 6)$. Si $u$ es una solución del problema $(1.1)-(1.3)$ sobre $\left[0, T_{\operatorname{máx}}\left[\right.\right.$ con datos iniciales $u_{0} \in H_{0}^{2}(\Omega)$ y $u_{1} \in L^{2}(\Omega)$, y satisfaciendo una de las siguientes condiciones:

(i) $E(0)<0$,

(ii) $E(0)=0$ y $A^{\prime}(0)>K_{0}$,

(iii) $0<E(0)<E_{0} \quad y \quad\left\|u_{0}\right\|>\lambda_{0}$,

(iv) $E_{0} \leq E(0)<\frac{\left[A^{\prime}(0)-K_{0}\right]^{2}}{8\left[A(0)+T_{1} K_{0}\right]}$ y $A^{\prime}(0)>r_{2}\left[A(0)+\frac{K_{1}}{4(\gamma+1)}\right]+K_{0}$, 
entonces $T_{\operatorname{máx}}<\infty$ y $\operatorname{lím}_{t \rightarrow T_{\max }^{-}}|u(t)|_{2}^{2}=\infty$. Además el tiempo finito $T_{\operatorname{máx}}$ es estimado, en el caso $(i)$,

$$
T_{\operatorname{máx}} \leq t_{0}-\frac{J\left(t_{0}\right)}{J^{\prime}\left(t_{0}\right)}
$$

Además, si $J\left(t_{0}\right) \leq \operatorname{mín}\left\{1, \sqrt{\frac{a_{1}}{-b_{1}}}\right\}$, se tiene

$$
T_{\text {máx }} \leq t_{0}+\frac{1}{\sqrt{-b_{1}}} \ln \left(\frac{\sqrt{\frac{a_{1}}{-b_{1}}}}{\sqrt{\frac{a_{1}}{-b_{1}}}-J\left(t_{0}\right)}\right) .
$$

En el caso (ii),

$$
T_{\text {máx }} \leq t_{0}-\frac{J\left(t_{0}\right)}{J^{\prime}\left(t_{0}\right)}
$$$$
T_{\operatorname{máx}} \leq t_{0}+\frac{J\left(t_{0}\right)}{\sqrt{a_{1}}} .
$$

En el caso (iii),

$$
T_{\operatorname{máx}} \leq t_{0}-\frac{J\left(t_{0}\right)}{J^{\prime}\left(t_{0}\right)}
$$

Además, si $J\left(t_{0}\right) \leq \operatorname{mín}\left\{1, \sqrt{\frac{a_{2}}{-b_{2}}}\right\}$, se obtiene

$$
T_{\text {máx }} \leq t_{0}+\frac{1}{\sqrt{-b_{2}}} \ln \left(\frac{\sqrt{\frac{a_{2}}{-b_{2}}}}{\sqrt{\frac{a_{2}}{-b_{2}}}-J\left(t_{0}\right)}\right) .
$$

En el caso $(i v)$,

$$
o
$$

$$
T_{\text {máx }} \leq \frac{J\left(t_{0}\right)}{\sqrt{a_{1}}}
$$

$$
T_{\operatorname{máx}} \leq t_{0}+2^{\frac{3 \gamma+1}{2 \gamma}} \frac{\gamma c}{\sqrt{a_{1}}}\left\{1-\left[1+c J\left(t_{0}\right)\right]^{-\frac{1}{2 \gamma}}\right\}
$$

donde

$a_{1}:=\gamma^{2}\left[J\left(t_{0}\right)\right]^{\frac{2}{\gamma}+2}\left[\left[A^{\prime}\left(t_{0}\right)-K_{0}\right]^{2}-8 E(0)\left[J\left(t_{0}\right)\right]^{\frac{-1}{\gamma}}\right], \quad b_{1}:=8 \gamma^{2} E(0), \quad c:=\left(\frac{b_{1}}{a_{1}}\right)^{\frac{\gamma}{\gamma+2}}, a_{2}:=$ $\gamma^{2}\left[J\left(t_{0}\right)\right]^{\frac{2}{\gamma}+2}\left[\left[A^{\prime}\left(t_{0}\right)-K_{0}\right]^{2}+\frac{2 K_{2}}{2 \gamma+1}\left[J\left(t_{0}\right)\right]^{\frac{-1}{\gamma}}\right]$ y $b_{2}:=-\frac{2 K_{2} \gamma^{2}}{2 \gamma+1}$.

En el caso $(i), t_{0}:=\operatorname{máx}\left\{\frac{A^{\prime}(0)-K_{0}}{4(1+2 \gamma) E(0)}, 0\right\}$, en los casos $(i i)$ y $(i v), t_{0}:=0$ y $t_{0}:=\operatorname{máx}\left\{\frac{K_{0}-A^{\prime}(0)}{K_{2}}, 0\right\}$ en el caso (iii).

Demostración. Por diferenciación de (5.20), resulta

$$
J^{\prime}(t)=-\gamma[J(t)]^{\frac{1}{\gamma}+1}\left[A^{\prime}(t)-K_{0}\right]
$$

y

$$
J^{\prime \prime}(t)=-\gamma[J(t)]^{\frac{2}{\gamma}+1} V(t)
$$

donde

$$
V(t):=A^{\prime \prime}(t)\left[A(t)+\left(T_{1}-t\right) K_{0}\right]-(\gamma+1)\left[A^{\prime}(t)-K_{0}\right]^{2} .
$$

Por $\left(\alpha_{1} \beta_{1}+\alpha_{2} \beta_{2}\right)^{2} \leq\left(\alpha_{1}^{2}+\alpha_{2}^{2}\right)\left(\beta_{1}^{2}+\beta_{2}^{2}\right)$, (5.17) y la desigualdad de Hölder, de (5.11), resulta

$$
\left[A^{\prime}(t)-K_{0}\right]^{2} \leq 4\left[A(t)+\left(T_{1}-t\right) K_{0}\right]\left[\left|u^{\prime}(t)\right|_{2}^{2}+\int_{0}^{t}\left|\sqrt{\beta} u^{\prime}(s)\right|_{2}^{2} d s\right] .
$$


Por (5.10) y (5.32), de (5.31) obtenemos

$$
\begin{aligned}
V(t) \geq & {\left[Q(t)+4(\gamma+1)\left[\left|u^{\prime}(t)\right|_{2}^{2}+\int_{0}^{t}\left|\sqrt{\beta} u^{\prime}(s)\right|_{2}^{2} d s\right]\right][J(t)]^{-\frac{1}{\gamma}} } \\
& -4(\gamma+1)\left[\left|u^{\prime}(t)\right|_{2}^{2}+\int_{0}^{t}\left|\sqrt{\beta} u^{\prime}(s)\right|_{2}^{2} d s\right][J(t)]^{-\frac{1}{\gamma}} \\
= & Q(t)[J(t)]^{-\frac{1}{\gamma}}, \text { para } t \geq t_{0} .5 .33
\end{aligned}
$$

Por (5.33), de (5.30) obtenemos

$$
J^{\prime \prime}(t) \leq-\gamma[J(t)]^{\frac{1}{\gamma}+1} Q(t), \text { para } t \geq t_{0} .
$$

Ahora analicemos de acuerdo al signo de la energía inicial $E(0)$. Para $E(0) \leq 0$, de (5.10) y (5.34), resulta

$$
J^{\prime \prime}(t) \leq 4 \gamma(2 \gamma+1) E(0)[J(t)]^{\frac{1}{\gamma}+1}, \text { para } t \geq t_{0} .
$$

De (5.14) y (5.29), se tiene

$$
J^{\prime}(t)<0, \text { para } t>t_{0} .
$$

Multiplicando (5.35) por $J^{\prime}(t)$ y luego integrando de $t_{0}$ a $t$, tenemos

$$
\left[J^{\prime}(t)\right]^{2} \geq a_{1}+b_{1}[J(t)]^{2+\frac{1}{\gamma}}, \text { para } t \geq t_{0}
$$

donde

$$
\begin{aligned}
a_{1} & :=\left[J^{\prime}\left(t_{0}\right)\right]^{2}-8 \gamma^{2} E(0)\left[J\left(t_{0}\right)\right]^{\frac{1}{\gamma}+2} \\
& =\gamma^{2}\left[J\left(t_{0}\right)\right]^{\frac{2}{\gamma}+2}\left[\left[A^{\prime}\left(t_{0}\right)-K_{0}\right]^{2}-8 E(0)\left[J\left(t_{0}\right)\right]^{\frac{-1}{\gamma}}\right]
\end{aligned}
$$

$\mathrm{y}$

$$
b_{1}:=8 \gamma^{2} E(0)
$$

Un caso particular cuando $E(0)<0$, por $(5.36)$ y $(5.37)$, se obtiene directamente $\lim _{t \rightarrow T_{*}^{-}} J(t)=0$ y la estimativa (5.21) para el tiempo finito $T_{*}$. Para las estimativas (5.22) y (5.24), por (5.36) y (5.37), la función $J$ satisface las condiciones del Lema 2.4. Entonces existe un tiempo finito $T_{*}$ tal que $\lim _{t \rightarrow T_{*}^{-}} J(t)=0$ y se tiene las estimativas (5.22) y (5.24). Obsevar que las estimativas (5.23) y (5.24) son equivalentes.

Para el caso $0<E(0)<E_{0}$, de (5.34) y (5.16), se consigue

$$
J^{\prime \prime}(t) \leq-\gamma K_{2}[J(t)]^{\frac{1}{\gamma}+1}, \text { para } t \geq t_{0} .
$$

Con los mismos argumentos que se obtuvo la relación (5.37), se obtiene

$$
\left[J^{\prime}(t)\right]^{2} \geq a_{2}+b_{2}[J(t)]^{2+\frac{1}{\gamma}}, \text { para } t \geq t_{0}
$$

donde

$$
a_{2}:=\gamma^{2}\left[J\left(t_{0}\right)\right]^{\frac{2}{\gamma}+2}\left[\left[A^{\prime}\left(t_{0}\right)-K_{0}\right]^{2}+\frac{2 K_{2}}{2 \gamma+1}\left[J\left(t_{0}\right)\right]^{\frac{-1}{\gamma}}\right]
$$

y

$$
b_{2}:=-\frac{2 K_{2} \gamma^{2}}{2 \gamma+1}
$$

Por (5.36) y (5.38), la función $J$ satisface las condiciones del Lema 2.4. Entonces existe un tiempo finito $T_{*}$ tal que $\lim _{t \rightarrow T_{*}^{-}} J(t)=0 \mathrm{y}$ se tiene la estimativa (5.26). La estimativa (5.25) se obtiene directamente de las relaciones (5.36) y (5.38).

Para el caso $E_{0} \leq E(0)$. Con los mismos argumentos para el caso $E(0) \leq 0$, se obtiene la relación (5.37). Observemos que 


$$
a_{1}>0 \text { si y solo si } E(0)<\frac{\left[A^{\prime}\left(t_{0}\right)-K_{0}\right]^{2}}{8\left[A\left(t_{0}\right)+\left(T_{1}-t_{0}\right) K_{0}\right]} .
$$

Nuevamente por (5.36) y (5.38), la función $J$ satisface las condiciones del Lema 2.4. Entonces existe un tiempo finito $T_{*}$ tal que $\lim _{t \rightarrow T_{*}^{-}} J(t)=0 \mathrm{y}$ se tiene las estimativas $(5.27)$ y (5.28).

Desde que $\left[0, T_{\text {máx }}[\right.$ es el intervalo maximal de las soluciones del problema (1.1) - (1.3), resulta que $T_{\text {máx }}=T_{*}$. También por $\lim _{t \rightarrow T_{\text {máx }}^{-}} J(t)=0$, se obtiene

$$
\lim _{t \rightarrow T_{\text {máx }}^{-}} A(t)=\infty
$$

De aquí, se deduce

$$
\lim _{t \rightarrow T_{\max }^{-}}|u(t)|_{2}^{2}=\infty .
$$

Con todo esto se concluye la demostración del Teorema 5.10.

Observación 5.11. La selección de $T_{1}$ de (5.20) es posible escoger con algunas consideraciones. Las discusiones son similares como en [13]. Omitimos los detalles.

\section{BIBLIOGRAFÍA}

[1] Adams R. A., Sobolev Space, Academic Press, New York, 1975.

[2] Ball J. M., Initial boundary value problem for an extensible beam, Journal of Mathematical Analysis and Application, 42 (1973), 61-90.

[3] Burgreen D., Free vibrations of a pin-ended column with constant distance between pin ends, J. Appl. Mech. 18 (1951), 135-139.

[4] Coddington E. A. and Levinson N., Theory of ordinary differential equations; McGraw-Hill, New York, 1955.

[5] Dickey R. W., Free vibrations and dynamic buckling of the extensible beam, J. Math. Anal. Appl. 29 (1970), 443-454.

[6] Eisley J. G., Nonlinear vibrations of beams and rectangular plates, Z. Angew. Math. Phys. 15 (1964), $167-175$.

[7] Guedda M. and Labani H., Nonexistence of global solutions to a class of nonlinear wave equations with dynamic boundary conditions, Bull. Belg. Math. Soc. 9 (2002), 39-46.

[8] Kirchhoff G., Vorlesungen über mechanik, Leipzig, Teubner, 1883.

[9] Li, M.-R. and Tsai L.-Y., On a system of nonlinear wave equation, Taiwanese Journal of Mathematic Vol.7, No. 4, pp. 557-573, December 2003.

[10] Quispe Méndez, T., Solución local de una ecuación de Kirchhoff no lineal viscoelástica con término disipativo, PESQUIMAT Revista de la Fac. CC. MM. de la Universidad Nacional Mayor de San Marcos Vol. X, No.1, pp 11-32, LIMA-PERÚ. Agosto 2007.

[11] Quispe Méndez, T., Singularidad de soluciones para una ecuación de Kirchhoff no lineal viscoelástica con término disipativo, PESQUIMAT Revista de la Fac. CC. MM. de la Universidad Nacional Mayor de San Marcos Vol. X, No.2, pp 67-80, LIMA-PERÚ. Noviembre 2007. 
[12] Quispe Méndez, T. y Carrillo Díaz, L. E., Solución local de un sistema de Kirchhoff no lineal viscoelástico con término disipativo, PESQUIMAT Revista de la Fac. CC. MM. de la Universidad Nacional Mayor de San Marcos Vol. XIII, No.2, pp 40-58, LIMA-PERÚ. Diciembre 2010.

[13] Quispe Méndez, T., Singularidad de soluciones para un sistema de Kirchhoff no lineal viscoelástico con término disipativo, por aparecer en PESQUIMAT Revista de la Fac. CC. MM. de la Universidad Nacional Mayor de San Marcos.

[14] Tucsnak M., Semi-internal stabilization for a nonliner Euler-Bernoulli equation, Mathematical Methods in the Applied Sciences, 19 (1996), 897-907.

[15] Vasconcellos C. F. and Teixeira L. M., Existence, uniqueness and stabilization for a nonlinear plate system with nonlinear damping, Ann. Fac. Sci. Toulouse Math., (6), 8, (1999), no. 1, pp. 173-193.

[16] Woinowsky-Krieger S., The effect of axial force on the vibration of hinged bars, J. Appl. Mech. 17 (1950), 35-36.

[17] Wu S.-T. and Tsai L.-Y., Existence and nonexistence of global solutions for a nonlinear wave equation, Taiwanese Journal of Mathematic Vol.13, No. 6B, pp. 2069-2091, December 2009.

[18] Zeidler E., Nonlinear functional analysis and its applications I: Fixed-point theorems, SpringerVerlag, New York, 1986. 